\title{
A case of monoclonal gammopathy of renal significance presenting as atypical amyloidosis with IgA lambda paraproteinemia
}

\author{
Chankyung Kim ${ }^{1}$, John Brealey, ${ }^{1}$ Anjelo Jobert ${ }^{2}$, James Nolan ${ }^{1}$ \\ 'Department of Anatomical Pathology, SA Pathology, Adelaide; \\ ${ }^{2}$ Central and Northern Adelaide Renal Transplantation Services, Royal Adelaide Hospital, Adelaide, Australia
}

\begin{abstract}
Monoclonal gammopathy of renal significance is defined as any B cell or plasma cell clonal lymphoproliferation which neither causes tumor complications nor meets any current hematological criteria for specific therapy, with one or more kidney lesions related to the produced monoclonal immunoglobulin, such as amyloidosis. A 50-year-old male presented with heavy proteinuria and blood tests showing IgA and Lambda paraproteinemia. Light microscopy showed mesangial eosinophilic ground substance extending into the capillary loops, and positive staining within the glomeruli and vessel walls for amyloid P immunohistochemistry was also noted. Immunofluorescence showed positive staining for IgA and Lambda in the mesangia and capillary loops. Electron microscopy exhibited organized fibrils measuring $4-5 \mathrm{~nm}$ in diameter in the mesangia, glomerular basement membranes and vessel walls. We interpreted the overall findings as atypical renal amyloidosis with IgA and Lambda deposition on immunofluorescence. Further amyloid typing using laser microdissection-liquid chromatography and mass spectrometry will be useful.
\end{abstract}

Key Words: Monoclonal gammopathy of renal significance; AHL amyloidosis; Immunoglobulin A; Atypical amyloidosis

Received: June 18, 2020 Revised: August 12, 2020 Accepted: September 18, 2020

Corresponding Author: James Nolan, MBBS, FRCPA, Department of Anatomical Pathology, SA Pathology, Royal Adelaide Hospital, Port Rd, Adelaide SA 5000, Australia

Tel: +61-8-7074-1181, Fax: +61-8-7074-6148, E-mail: James.Nolan@sa.gov.au

Amyloid is a material that is deposited in vivo that displays a characteristic fibrillary electron microscopic appearance and an affinity for Congo red stain, with green birefringence on polarized light microscopy [1]. Amyloidosis is a disease secondary to amyloid deposition. There are a range of amyloid proteins among which monoclonal kappa/lambda immunoglobulin light chain (AL) and AA are commonly associated with renal involvement. Moreover, other amyloid proteins have been reported to involve the kidney, including TR, AAPO A1, AAPO A2, Afib, ALys, AGel, ACysC and ALECT2 [2].

The International Kidney and Monoclonal Gammopathy Research Group (IKMG) defines monoclonal gammopathy of renal significance (MGRS) as any B cell or plasma cell clonal lymphoproliferation with both of the following characteristics: (1) one or more kidney lesions that are related to the produced monoclonal immunoglobulin and (2) the underlying B cell or plasma cell clone does not cause tumor complications or meet any current hematological criteria for specific therapy [3]. There are various renal diseases associated with MGRS, and they are now known as MGRS-associated lesions, conditions or disorders [3].

\section{CASE REPORT}

A 50-year-old male was evaluated by a renal physician due to the clinical features of nephrotic syndrome including heavy proteinuria and edema. A native kidney biopsy was sent to our laboratory for light microscopy, fresh frozen immunofluorescence and electron microscopy. Blood tests showed albumin of $17 \mathrm{~g} / \mathrm{L}$ (normal range, 34 to $48 \mathrm{~g} / \mathrm{L}$ ) and creatinine of $99 \mu \mathrm{mol} /$ $\mathrm{L}$ (normal range, 60 to $110 \mu \mathrm{mol} / \mathrm{L}$ ), and the 24-hour urine protein was $8,596 \mathrm{mg} / 24 \mathrm{hr}$. The serum free kappa/lambda light chain ratio measured 0.09 (kappa light chain $=24.03 \mathrm{mg} / \mathrm{L}$ [normal range, 3.00 to $19.00 \mathrm{mg} / \mathrm{L}$ ], lambda light chain = $276.87 \mathrm{mg} / \mathrm{L}$ [normal range, 6.00 to $26.00 \mathrm{mg} / \mathrm{L}$ ]). The serum electrophoresis detected $5 \mathrm{~g} / \mathrm{L}$ of paraprotein of IgA lambda and free lambda types.

A bone marrow biopsy showed the following: mildly increased plasma cells, less than 5\%; phenotype: $\mathrm{CD} 38^{+}, \mathrm{CD} 138^{+}, \mathrm{CD} 19^{-}$, 

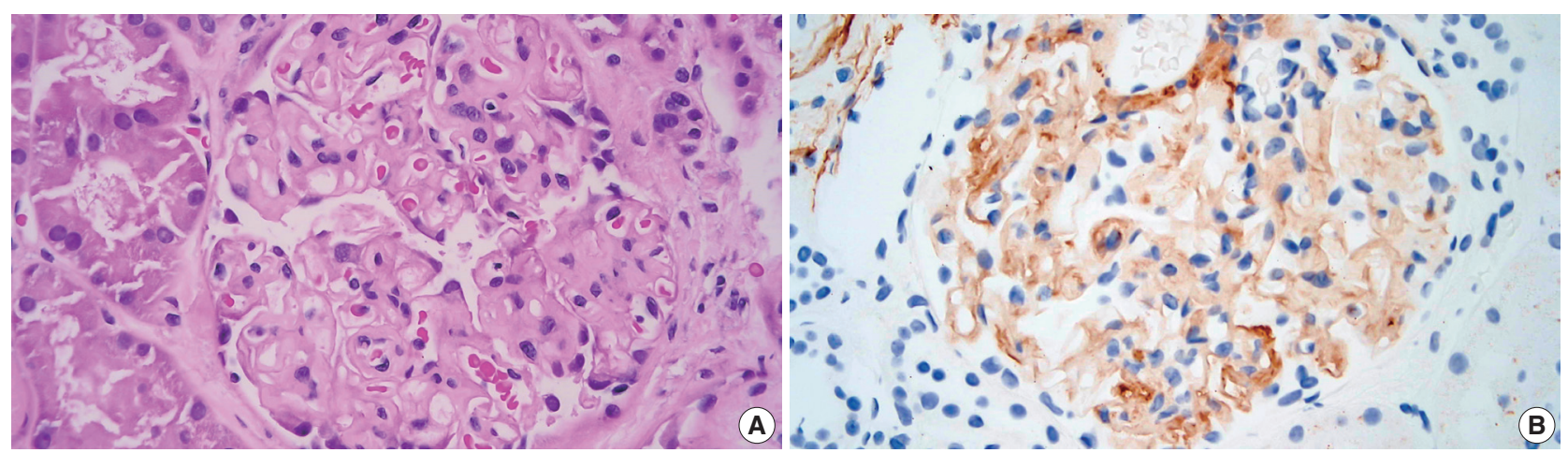

Fig. 1. Hematoxylin and eosin stain and Amyloid P immunohistochemistry. (A) The glomerulus shows mesangial eosinophilic ground substance which extends into the capillary loops. (B) Positive staining is seen within the glomerulus with amyloid P immunohistochemistry.
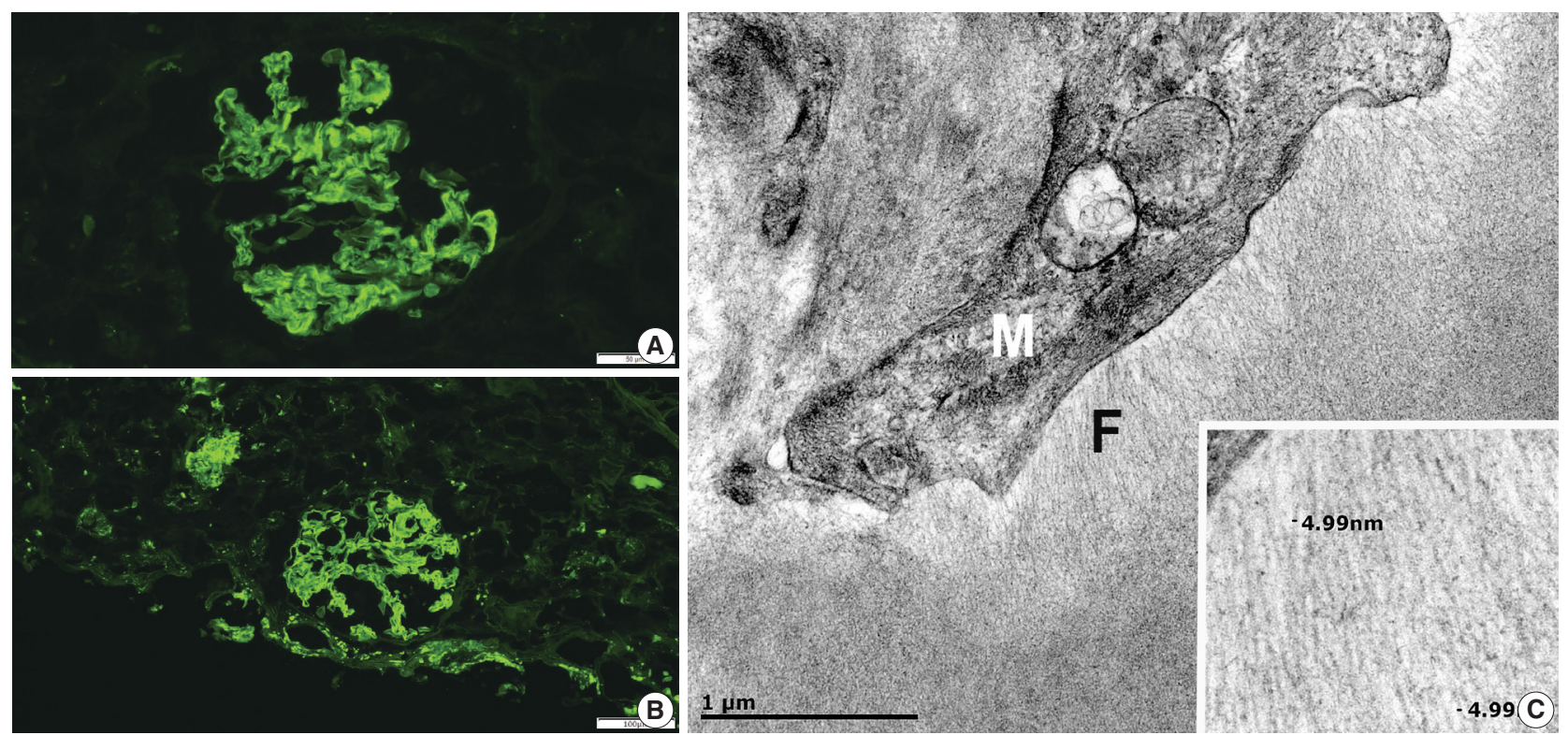

Fig. 2. Immunofluorescence and electron microscopy. (A) Positive mesangial and capillary loop staining with IgA on immunofluorescence. (B) Positive mesangial and capillary loops staining with Lambda on immunofluorescence. (C) The fine fibrillary material (F) is present adjacent to the mesangial cell $(M)(\times 40,000)$. The inset shows amyloid-like fibrils measuring approximately $5 \mathrm{~nm}$ in diameter $(\times 120,000)$.

$\mathrm{CD}^{-} 0^{-}, \mathrm{CD} 56^{-}, \mathrm{CD} 28^{+}, \mathrm{CD}_{117}^{-}$; and cytoplasmic lambda light chain restriction. This was consistent with monoclonal gammopathy of undetermined significance (MGUS).

A percutaneous core renal biopsy was performed, and the following tissues were submitted: formalin-fixed tissue for histology, fresh tissue for immunofluorescence and glutaraldehydefixed tissue for electron microscopy.

Light microscopy showed mesangial eosinophilic ground substance extending into the capillary loops (Fig. 1A). Positive staining within the glomeruli and vessel walls was identified for amyloid P immunohistochemistry (Fig. 1B). The Congo red stain demonstrated faint non-specific staining of the glomeruli in which no green birefringence was seen on polarized light microscopy.
Immunofluorescence showed positive staining for IgA (Fig. 2A) and Lambda (Fig. 2B) in the mesangia and capillary loops. Electron microscopy exhibited fibrils measuring 4-5 $\mathrm{nm}$ in diameter in the mesangia, glomerular basement membranes and vessel walls (Fig. 2C).

\section{DISCUSSION}

We interpret this case as an unusual form of amyloidosis in a patient with a clinical presentation of heavy proteinuria in the setting of MGUS. The overall findings fit with a recently described diagnostic entity known as MGRS. Deposition of fibrillary eosinophilic material that show positive labelling with amyloid P im- 
munohistochemistry in the mesangia, glomerular basement membranes and blood vessels supports the diagnosis of amyloidosis. On the other hand, there were two factors that are unusual for typical amyloidosis. First, the fibrils in this case only measured 4-5 $\mathrm{nm}$ in thickness, whereas amyloid fibrils typically measure 7-12 nm [4]. The absence of green birefringence or anomalous colors on polarized microscopy was the second unusual finding.

The weak non-specific staining of glomeruli with Congo red without green birefringence on polarized microscopy observed in this case could be secondary to the properties of the amyloid protein. A study by Suhr et al. [5] revealed that different types of transthyretin (TTR) amyloid fibrils can exhibit altered affinity for Congo red stain and birefringence. For example, type A, which consists of carboxy-terminal TTR-related amyloidosis (ATTR) fragments and full-length TTR, displayed strong affinity for Congo red stain, whereas type $\mathrm{B}$, which consists only of full-length TTR, showed weak affinity for Congo red stain and absence of glittering birefringence. Thus, amyloids may demonstrate a variable affinity for Congo red and variable birefringence.

The immunofluorescence staining suggests that the amyloid is of IgA lambda origin, and hence, a diagnosis of IgA-lambda type renal heavy- and light-chain amyloidosis (AHL amyloidosis) is being considered. However, there is a case report from Japan which described AL amyloidosis in a patient with lymphoplasmacytic lymphoma secreting IgM-kappa chains [6]. In this case, the origin of the amyloid was initially thought to be from both IgM heavy and kappa light chain as suggested by renal immunofluorescence and laser microdissection-liquid chromatography and mass spectrometry (LMD-LC-MS). However, LMDLC-MS of the amyloid fibrils after the amyloid purification method suggested that the heavy chain identified was actually from the non-specific co-deposition of monoclonal IgM-kappa, which demonstrated that the amyloid was only of light chain origin. Therefore, it would be imprudent to diagnose the present case as AHL amyloidosis until we obtain the LMD-LC-MS results.

According to the study published by Nasr et al. [7], among 16 heavy-chain $(\mathrm{AH})$ and heavy- and light-chain renal amyloidosis cases, five showed detection of $\operatorname{IgA}$, and one showed both $\operatorname{IgA}$ and lambda positivity on laser microdissection and mass spectrometry (LMD-MS). There were 202 cases of AL renal amyloidosis during the same time period, with $\mathrm{AH} / \mathrm{AHL}$ renal amyloidosis cases accounting for $7.3 \%$ of the entire cohort and $\operatorname{IgA}$ and lambda positive $\mathrm{AH} / \mathrm{AHL}$ occupying only less than $0.01 \%$ of the entire cohort. Interestingly, there was a case in which immunofluorescence was positive for both $\operatorname{IgA}$ and lambda, but only $\operatorname{IgA}$ was found on LMD-MS. This again demonstrates the difficulty in accurate typing of AH/AHL solely by immunofluorescence.

Given the unusual findings for typical amyloidosis in the present case, other possible diagnoses were considered. Negative staining with Congo red suggested monoclonal immunoglobulin deposition disease (MIDD) as an alternate diagnosis, especially when amyloid P immunohistochemistry can sometimes show non-specific staining (from our experiences). However, electron microscopy showed organized fibrils rather than the non-fibrillar "powdery" electron dense deposits which are typically seen in MIDD [8]. This made the diagnosis of MIDD less likely. Positive $\operatorname{IgA}$ staining on immunofluorescence is unusual for amyloidosis and raised the possibility of IgA nephropathy, but no mesangial or endocapillary hypercellularity was present, $C 3$ was negative, and most importantly, the clinical presentation did not fit with IgA nephropathy. Hence, this was disregarded from our diagnostic work-up.

As mentioned, further renal amyloid typing can be performed by LMD-LC-MS [3]. Vrana et al. [9] reported that this method showed $100 \%$ specificity and $98 \%$ sensitivity in the identification of the types of amyloid protein. This may be indicated in our case due to the questionable Congo red staining and bright staining of IgA by immunofluorescence. Identification of the amyloid type is incredibly important as the disease management depends on the underlying cause of amyloidosis. In our case, further amyloid typing is being considered, and meanwhile, the patient will undergo autologous bone marrow transplantation for treatment.

\section{Ethics Statement}

Informed consent was obtained from the patient, and the study was approved by the Central Adelaide Local Health Network Human Research Ethics Committee (CALHN reference number: 13298, approved on 01/06/2020)

\section{ORCID}

Chankyung Kim https://orcid.org/0000-0001-6054-0605 James Nolan https://orcid.org/0000-0002-6034-7159

\section{Author Contributions}

Conceptualization: CK, JN. Data curation: CK. Formal analysis: CK, JB, JN. Investigation: CK, JN. Methodology: CK, JB, AJ, JN. Resources: JB, AJ, JN. Supervision: JN. Validation: JB, AJ, JN. Visualization: CK. Writingoriginal draft: CK. Writing—review \& editing: CK, JB, AJ, JN. Approval of final manuscript: all authors.

\section{Conflicts of Interest}

The authors declare that they have no potential conflicts of interest.

\section{Funding Statement}

No funding to declare. 


\section{Acknowledgments}

We would like to thank Associate Professor Sonja Klebe from anatomical pathology department of Flinders Medical Centre and Dr Sarita Prabhakaran from Flinders University for their assistance in photo editing and guidance in obtaining ethics approval.

\section{References}

1. Westermark P, Benson MD, Buxbaum JN, et al. A primer of amyloid nomenclature. Amyloid 2007; 14: 179-83.

2. Holanda DG, Acharya VK, Dogan A, Racusen LC, Atta MG. Atypical presentation of atypical amyloid. Nephrol Dial Transplant 2011; 26: 373-6.

3. Leung N, Bridoux F, Batuman V, et al. The evaluation of monoclonal gammopathy of renal significance: a consensus report of the International Kidney and Monoclonal Gammopathy Research Group. Nat Rev Nephrol 2019; 15: 45-59.

4. Leung N, Drosou ME, Nasr SH. Dysproteinemias and glomerular disease. Clin J Am Soc Nephrol 2018; 13: 128-39.
5. Suhr OB, Lundgren E, Westermark P. One mutation, two distinct disease variants: unravelling the impact of transthyretin amyloid fibril composition. J Intern Med 2017; 281: 337-47.

6. Manabe S, Iwasaki C, Hatano M, et al. AL amyloidosis with nonamyloid forming monoclonal immunoglobulin deposition: a case mimicking AHL amyloidosis. BMC Nephrol 2018; 19: 337.

7. Nasr SH, Said SM, Valeri AM, et al. The diagnosis and characteristics of renal heavy-chain and heavy/light-chain amyloidosis and their comparison with renal light-chain amyloidosis. Kidney Int 2013; 83: 463-70.

8. Nasr SH, Valeri AM, Cornell LD, et al. Renal monoclonal immunoglobulin deposition disease: a report of 64 patients from a single institution. Clin J Am Soc Nephrol 2012; 7: 231-9.

9. Vrana JA, Gamez JD, Madden BJ, Theis JD, Bergen HR 3rd, Dogan A. Classification of amyloidosis by laser microdissection and mass spectrometry-based proteomic analysis in clinical biopsy specimens. Blood 2009; 114: 4957-9. 